\title{
Lamp2a is required for tumor growth and promotes tumor recurrence of hepatocellular carcinoma
}

\author{
ZHEN-BIN DING $^{1 *}$, XIU-TAO FU ${ }^{1 *}$, YING-HONG SHI $^{1 *}$, JIAN ZHOU $^{1,2^{*}}$, YUAN-FEI PENG $^{1}$, \\ WEI-REN LIU ${ }^{1}$, GUO-MING SHI ${ }^{1}$, QIANG GAO ${ }^{1}$, XIAO-YING WANG ${ }^{1}$, KANG SONG ${ }^{1}$, \\ LEI JIN ${ }^{1}$, MENG-XIN TIAN ${ }^{1}$, YING-HAO SHEN ${ }^{1}$ and JIA FAN ${ }^{1,2}$ \\ ${ }^{1}$ Liver Cancer Institute, Zhongshan Hospital, and Key Laboratory of Carcinogenesis and Cancer Invasion \\ (Ministry of Education); ${ }^{2}$ Institute of Biomedical Sciences, Fudan University, Shanghai 200032, P.R. China
}

Received July 27, 2016; Accepted September 20, 2016

DOI: 10.3892/ijo.2016.3754

\begin{abstract}
Exploring the function of chaperone-mediated autophagy (CMA) in cancer has promoted progress in cancer treatment through the regulation of CMA pathways. However, CMA status and function in hepatocellular carcinoma (HCC) by focusing on the regulatory role of lysosome-associated membrane protein type 2a (Lamp2a) remain to be clarified. We examined Lamp2a in a normal human liver cell line, 6 HCC cell lines, 10 normal liver samples as well as 42 HCC tissue and para-tumor tissues samples, and then validated it in 228 HCC patients to assess the relationship between Lamp2a and clinical prognosis. Gain and loss of Lamp2a function were also explored in HCC cell lines and xenograft models. Significantly lower level of Lamp2a expression was found in HCC cells and tissues compared with normal hepatic cells, para-tumor tissues and normal livers. Although no differences in HCC cell morphology or function were observed in relation to Lamp2a expression under normal culture or shortterm starvation conditions, Lamp2a blockage significantly inhibited HCC cell viability under prolonged starvation. Critically, Lamp2a is required for HCC xenograft growth in vivo by helping cells to avoid apoptosis and promoting cell proliferation. Furthermore, a significant correlation between
\end{abstract}

Correspondence to: Dr Jia Fan, Department of Liver Surgery, Zhongshan Hospital, Fudan University, 1609 Xietu Road, Shanghai 200032, P.R. China

E-mail: fan.jia@zs-hospital.sh.cn

*Contributed equally

Abbreviations: HCC, hepatocellular carcinoma; Lamp2a, lysosome-associated membrane protein type 2a; DMEM, Dulbecco's modified Eagle's media; HSC70/90, heat shock cognate 70/90-kDa protein; MTT, 3-(4,5-dimethyl-2-thiazolyl)-2,5-diphenyl-2-H-tetrazolium bromide; PCR, real-time polymerase chain reaction; TUNEL, terminal deoxynucleotidyl transferase-mediated deoxyuridine triphosphate nick-end labeling; TMA, tissue microarray

Key words: hepatocellular carcinoma, Lamp2a, chaperonemediated autophagy, tumor recurrence, cell viability
Lamp2a expression and tumor size or cumulative recurrence was uncovered in HCC patients. Collectively, the present study shows that impaired Lamp2a expression in HCC contributes to tumor cell viability and promotes tumor growth and recurrence. Targeting chaperone-mediated autophagy through Lamp2a may also imply a potentially novel treatment strategy for HCC.

\section{Introduction}

Hepatocellular carcinoma (HCC) is a major cause of cancer-related death (1). It is highly resistant to available chemotherapeutic agents, leaving HCC patients with no effective therapeutic option and a poor prognosis. The ability to avoid cell death is a hallmark of cancer cells, and their survival in an adverse environment under cellular stresses (e.g., hypoxia, nutrient deficiency and treatment stress) contributes to therapeutic failure and tumor progression $(2,3)$. Although cell death has been attributed to unrestrained autophagy, increasing data indicate that autophagy may also function as an important tumor-protective mechanism in $\mathrm{HCC}(4,5)$.

Chaperone-mediated autophagy (CMA) refers to selective autophagy of a subset of cytosolic proteins containing amino acid sequence motif KFERQ in lysosomes. Around $30 \%$ oxidatively modified proteins targeted to lysosomes by this pathway, and directly transport through the lysosome membrane for degradation (6). Modulation of CMA is known to underlie the pathogenesis of some systemic diseases such as Parkinson's disease (7), Danon disease (8) and mucolipidosis type IV (9). Therefore, understanding of CMA activities under different pathological and physiological conditions is increasing day by day, especially in cancer (10). Although in most cells a basal level of CMA activity may exist, the pathway is further activated by nutritional stress (11). Activation during prolonged starvation is associated with increased levels of lysosome-associated membrane protein type $2 \mathrm{a}$ (Lamp2a) at the lysosomal membrane, which represents a limiting step in this pathway $(10,12)$. Lamp2a in the lysosomal membrane acts as a receptor of substrate proteins for CMA, and Lamp2a in the lysosomal membrane have been shown to correlate directly with CMA activity under pathological and physiological conditions (13-15). 
We previously demonstrated that macroautophagy defects at an early stage of oncogenesis may contribute to the malignant differentiation and invasive phenotype in HCC (16). Furthermore, once a tumor is formed, macroautophagy can protect HCC cells against apoptosis induced by antineoplastic agents through its degradative process (17-19). We further investigated CMA status and function in HCC by focusing on the regulatory role of Lamp2a during cancer cell starvation.

\section{Materials and methods}

Cell lines and animals. The human HCC cell lines HepG2, Hep3B, Huh7, MHCC97L, MHCC97H and HCCLM3, and the human normal hepatic cell line L- 02 , were routinely maintained in high-glucose Dulbecco's modified Eagle's media (DMEM) supplemented with $10 \%$ heat-inactivated fetal bovine serum (FBS), $100 \mathrm{U} / \mathrm{ml}$ penicillin and $100 \mathrm{mg} / \mathrm{ml}$ streptomycin. All cell lines were cultured at $37^{\circ} \mathrm{C}$ in a humidified incubator in an atmosphere of $5 \% \mathrm{CO}_{2}$. Male athymic $\mathrm{BALB} / \mathrm{c}$ nude mice (4-weeks old; Shanghai Institute of Material Medicine, Chinese Academy of Science, Shanghai, China) were raised under pathogen-free conditions. All animal care and experimental protocols were carried out in accordance with the guidelines established by the Shanghai Medical Experimental Animal Care Commission.

Patient samples. Patient samples were collected after obtaining informed consent according to an established protocol approved by the Ethics Committee of Fudan University. The data did not contain any information that could lead to the identification of the individual patients.

Samples for real-time polymerase chain reaction (PCR) studies were randomly collected from patients undergoing curative resection for HCC at the Liver Cancer Institute, Zhongshan Hospital, Fudan University, in March 2006. Samples were collected immediately after resection, transported in liquid nitrogen, and stored at $-80^{\circ} \mathrm{C}$. Sixteen frozen tissue samples were also obtained from the above patients for western blot studies.

Tumor specimens for tissue microarray (TMA) studies were obtained from 228 consecutive HCC patients who underwent curative resection without preoperative treatment at the Liver Cancer Institute, Zhongshan Hospital, Fudan University, between 2005 and 2006. Complete follow-up data were available for each patient, and the diagnosis of HCC was confirmed by pathological examination.

Real-time PCR. Total RNA was extracted from cell lines and frozen tumor specimens using TRIzol reagent (Invitrogen, Carlsbad, CA, USA). Total RNA $(2 \mu \mathrm{g})$ was reverse transcribed using a RevertAid First-Strand cDNA Synthesis kit (Fermentas, Burlington, ON, Canada). Reverse transcription-PCR was performed before quantitative real-time PCR. Lamp2a mRNA expression was determined by real-time PCR using SYBR Premix Ex Taq (Takara Bio, Dalian, China). PCR amplification cycles were carried out for $10 \mathrm{sec}$ at $95^{\circ} \mathrm{C}$, followed by 40 cycles of $95^{\circ} \mathrm{C}$ for $5 \mathrm{sec}$ and $60^{\circ} \mathrm{C}$ for $30 \mathrm{sec}$. Data were collected after each annealing step. $\beta$-actin was used as an endogenous control to normalize for differences in the amounts of total RNA in each sample. Relative gene expression levels were calculated and were expressed as $2^{-\Delta \mathrm{Ct}}$, as previously described (16). The following primers were used: $\beta$-actin 5'-CAACTGGGACGACATGGAGAAAAT-3' and 5'-CCAG AGGCGTACAGGGATAGCAC-3'; Lamp2a 5'-AGACTGCA GTGCAGATGACGAC-3' and 5'-GACCAATAAAATAAGC CAGCAAC-3'.

Western blot analysis. Western blot analysis was performed as previously described (16). Briefly, proteins from total cell lysates were separated by standard sodium dodecyl sulfatepolyacrylamide gel electrophoresis and then transferred to polyvinylidene difluoride membranes. The membranes were washed, blocked and incubated with specific primary antihuman antibodies against $\beta$-actin (1:1,000; ab8226; Abcam, Cambridge, MA, USA) or Lamp2a (1:1,000; ab18528; Abcam), followed by incubation with horseradish peroxidase-conjugated secondary antibodies. The reactions were detected by enhanced chemiluminescence assay.

Transfection and clone selection. Lentiviral-mediated pGCGFP-Lamp2a and pGCSIL-GFP-Lamp2a short hairpin RNAs (shRNAs) were constructed (Shanghai Genechem, Co., Ltd., Shanghai, China). The shRNA targeting sequence (5'-AAGC ACCATCATGCTGGATAT-3') for Lamp2a was used. Huh7 and HCCLM3 cells were transfected with lentivirus particles and the cell populations expressing GFP-Lamp2a or GFP-Lamp2a shRNA were isolated by flow cytometry (BD Biosciences, San Jose, CA, USA). Stable transfectant clones were further validated by real-time PCR, and immunoblotting was used to determine the expression levels of Lamp2a protein.

Cell viability, migration and invasion assays. Cell viability was assessed using an MTT (3-(4,5-dimethyl-2-thiazolyl)2,5-diphenyl-2-H-tetrazolium bromide) kit (Trevigen, Gaithersburg, MD, USA), according to the manufacturer's protocol. Cells $\left(5 \times 10^{3}\right)$ were seeded in 96 -well plates, incubated for $24 \mathrm{~h}$ at $37^{\circ} \mathrm{C}$, and treated with the specified agents at defined time-points.

Cell migration was evaluated using the scratch-wound assay. Cells were cultured for 2 days to form a tight cell monolayer and then serum-starved for $6 \mathrm{~h}$. After serum starvation, the cell monolayer was wounded using a $20-\mu 1$ plastic pipette tip. The remaining cells were washed twice with culture medium to remove cell debris and incubated at $37^{\circ} \mathrm{C}$ with normal serum-containing culture medium. Migrating cells at the wound front were photographed at the indicated times, using an inverted microscope (Leica Microsystems, Wetzlar, Germany). The percentage of the cleared area at each time-point compared with time zero was measured using Image-Pro Plus software v6.2.

Cell invasion assays were performed using Transwell filters. Filters coated with Matrigel (BD Biosciences) in the upper compartment were loaded with $100 \mu 1$ medium containing $1 \times 10^{5}$ cells and the lower compartment was filled with conditioned culture medium mixed with DMEM and supplemented with 10\% FBS, NIH3T3 and HCC cell super supplements. After $36 \mathrm{~h}$, migrated cells on the bottom surface were fixed with $4 \%$ paraformaldehyde and counted after Giemsa staining. 
Autophagy analysis. Autophagy was assessed using GFP-LC3 redistribution. Redistribution of GFP-LC3 was detected using an inverted fluorescence microscope. The number of GFP-LC3-positive dots per cell was determined in three independent experiments. Eight randomly selected fields representing 200 cells were counted.

In vivo tumorigenicity. HCCLM3-control, HCCLM3-Lamp2a, Huh7-control and Huh7-Lamp2a shRNA cells $\left(5 \times 10^{6}\right)$ were suspended in $100 \mu \mathrm{l}$ serum-free DMEM and Matrigel (BD Biosciences) (1:1), and then inoculated into the liver parenchyma or the right upper flank subcutaneous region of nude mice, as previously described (20). The mice were sacrificed 5 weeks after tumor implantation. At necropsy, the volumes of the largest (a) and smallest (b) tumors were measured and the tumor volume was calculated as: $\mathrm{V}=\mathrm{ax} \mathrm{b}^{2} \mathrm{x} \pi / 6$. The tumor sections were prepared for immunohistochemical staining. Immunoreactivity was analyzed using Ki-67 (1:1,000; ab15580; Abcam) and P62 (1:1,000; ab56416; Abcam) in tumor tissues. Terminal deoxynucleotidyl transferase-mediated deoxyuridine triphosphate nick-end labeling (TUNEL) staining was performed using an In Situ Apoptosis Detection kit (VB-4005; GeneCopoeia, Rockville, MD, USA), according to the manufacturer's instructions.

TMA and immunohistochemistry. TMA was constructed as previously described (21). Briefly, all the HCC tissues were reviewed by two histopathologists, and representative areas free from necrotic and hemorrhagic material were premarked in the paraffin blocks. Two core biopsies (1 $\mathrm{mm}$ in diameter) were taken from the donor blocks and transferred to the recipient paraffin block at defined array positions. Three different TMA blocks were constructed, each containing 200 cylinders. Consecutive sections ( $4 \mu \mathrm{m}$ in thickness) were placed on 3-aminopropyltriethoxysilane-coated slides (Shanghai Biochip, Co., Ltd., Shanghai, China).

Immunohistochemistry was performed with monoclonal rabbit antibodies against human Lamp2a (1:100; ab18528; Abcam), using a two-step protocol (Novolink Polymer Detection system; Novocastra/Leica Biosystems, Richmond, IL, USA), as previously described (21). Briefly, after microwave antigen retrieval, tissues were incubated with primary antibodies for $60 \mathrm{~min}$ at room temperature, followed by incubation for $30 \mathrm{~min}$ with the secondary antibody (RE7112; Novolink Polymer; Leica Microsystems GmbH, Wetzlar, Germany). The sections were developed in 3,3'-diaminobenzidine solution under microscopic observation and counterstained with hematoxylin. Negative control slides, in which the primary antibodies were omitted, were included in all assays.

Evaluation of immunohistochemical variables. Immunohistochemical staining was evaluated by 3 independent pathologists with no knowledge of the patient characteristics. Scores were assigned for the intensity and percentage of positive staining in the cytoplasm in the whole cylinder. Discrepancies were resolved by consensus among the three pathologists, using a multihead microscope. The criteria for Lamp2a-positivity included moderate or strong immunoreactivity in $>20 \%$ of the cells. In the event of a difference between duplicate tissue cores, the higher score was considered to be the final score.
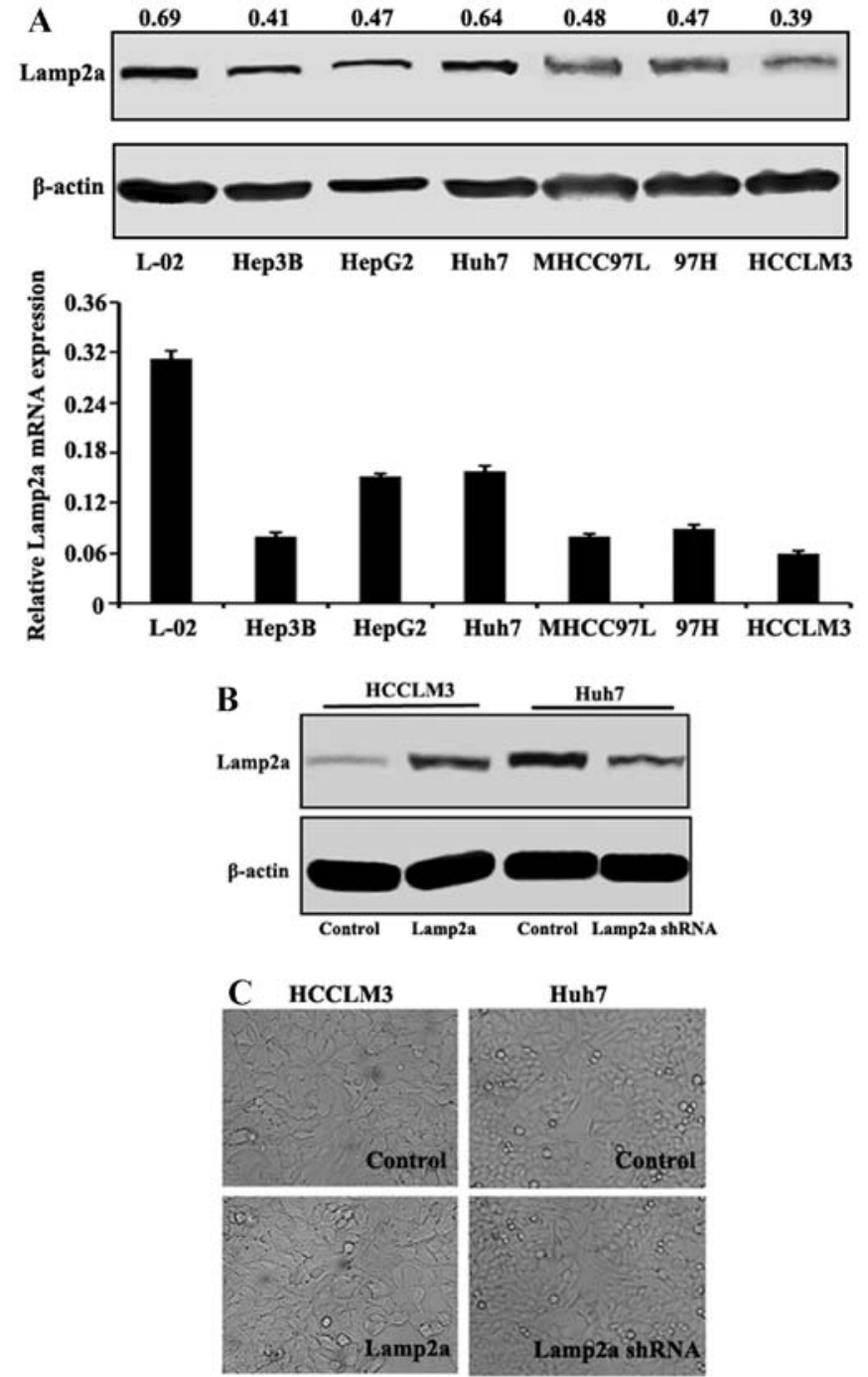

Figure 1. Lamp2a expression in HCC cell lines. (A) Expression of Lamp2a mRNA and protein was compared among a normal hepatic cell line L-02 and 6 established HCC cell lines with different malignant phenotype and invasive potentials. (B) Lamp2a expression in HCCLM3 and Huh7 modified by cDNA transfection or RNA interference was validated by western blotting. (C) Cell morphology of HCCLM3 and Huh7 modified by Lamp2a expression were observed under light microscopy. Magnification, x200.

Statistical analyses. Means were compared between two groups using unpaired, two-tailed Student's t-test, and among multiple groups using one-way analysis of variance. Categorical data were analyzed using $\chi^{2}$ or Fisher's exact tests. The Kaplan-Meier method was used to determine survival probability and differences were assessed by the log-rank test. Statistical significance was set at $\mathrm{P}<0.05$. All analyses were performed using SPSS software (v.16.0).

\section{Results}

Lamp2a expression in HCC cell lines. In several HCC cell lines with different malignant phenotypes and invasive potentials as previously described (16), Lamp2a mRNA and protein expression levels were evaluated. Lamp2a mRNA expression levels were lower in HCC cells compared with normal hepatic L-02 cells, as confirmed at the protein level by western blotting (Fig. 1A). Among the HCC cell lines, Lamp2a expression levels 

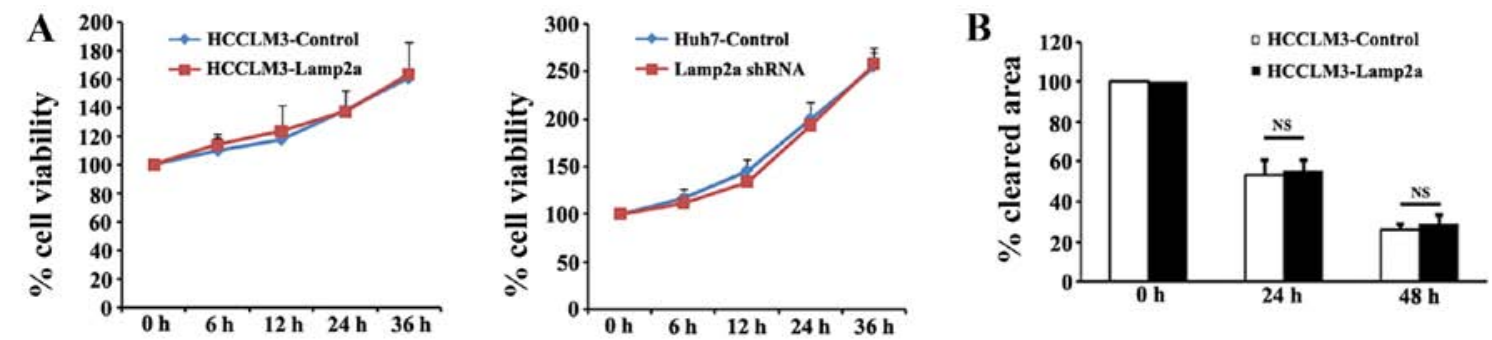

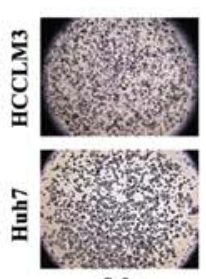

$\mathbf{0 ~ h}$

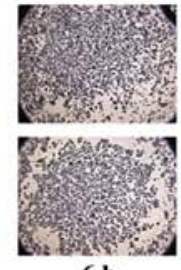

$6 \mathrm{~h}$

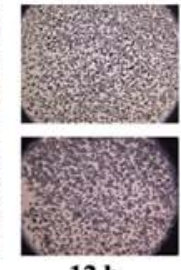

$12 \mathrm{~h}$



$24 \mathrm{~h}$

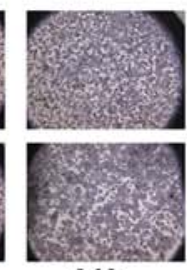

$36 \mathrm{~h}$
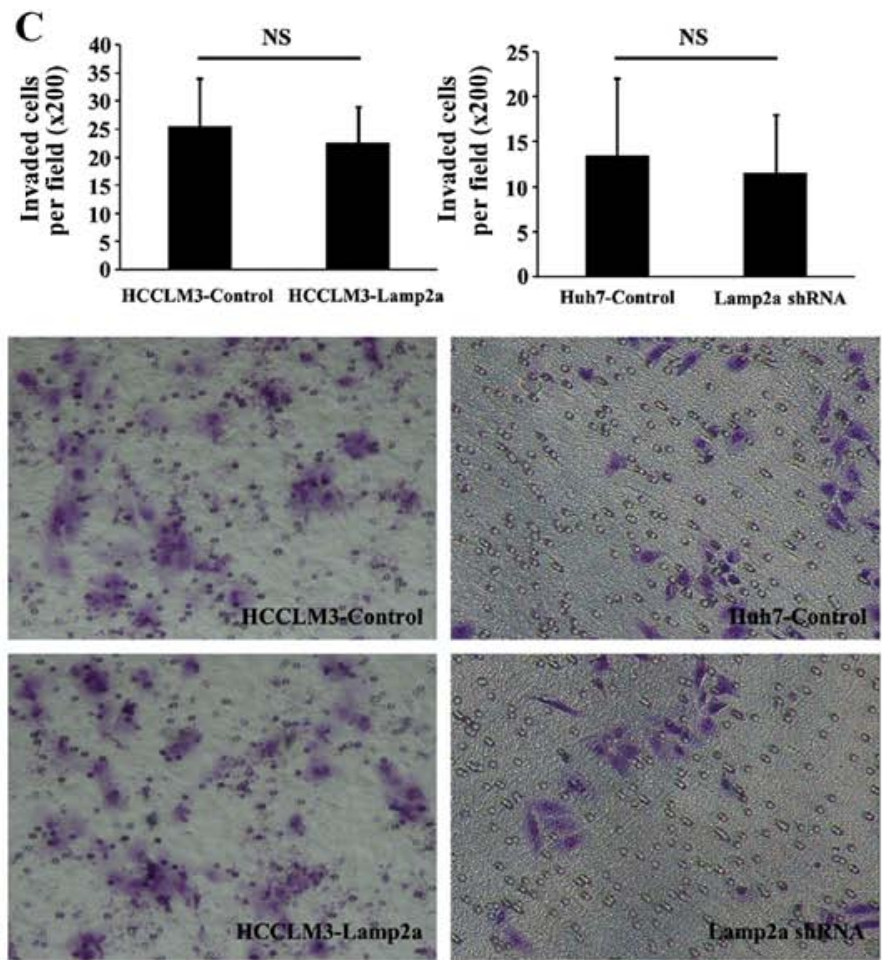

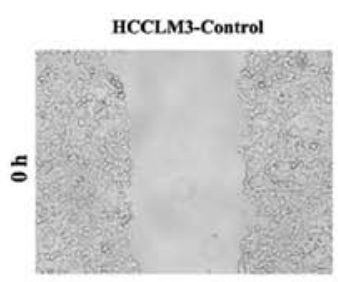

HCCLM3-Lamp2a


Figure 2. Cellular viability, migration and invasion in Lamp2a-modified HCCLM3 and Huh7 cell lines. (A) HCC cell line pairs (HCCLM3-Control/HCCLM3Lamp2a and Huh7-Control/Huh7-Lamp2a shRNA) were incubated in normal condition for 6-36 h, measured by absorbance at $570 \mathrm{~nm}$, and plotted as the percentage of the original time-point $(0 \mathrm{~h})$. Data shown are mean (SD) from 3 independent experiments. (B) Monolayer of HCC cells was wounded and then monitored at 0,24 and $48 \mathrm{~h}$ for wound channel closure. The cleared area was measured and plotted as the percentage of the original time-point $(0 \mathrm{~h})$. Data shown are mean (SD) from 3 independent experiments. Magnification, x200. NS, not significant. (C) Migration of HCC cells through a Matrigel-coated $8 \mu \mathrm{m}$ Transwell. After $36 \mathrm{~h}$, migrated cells on the bottom surface were fixed and counted after staining with Giemsa. Data shown are mean (SD) from 3 independent experiments. Twelve random fields were counted. Magnification, x200. NS, not significant.

were high in Huh7, intermediate in MHCC97L, MHCC97H and HepG2, and low in Hep3B and HCCLM3 cells, which did not correlate with their malignancy or invasiveness. We further evaluated the effect of Lamp2a expression in HCC cells in paired isogenic HCC cell lines (termed HCCLM3Control/HCCLM3-Lamp2a and Huh7-Control/Huh7-Lamp2a shRNA) in which Lamp2a expression was modified by RNA interference or cDNA transfection (Fig. 1B). Light microscopy showed no significant difference in cell morphology in relation to Lamp2a expression (Fig. 1C).
We also assessed cellular viability, migration ability and invasion ability in paired HCC cell lines. Two groups of HCC cells were incubated under normal conditions for $6-36 \mathrm{~h}$ and cellular viability was measured using MTT assays. There was no significant difference in survival in cells with upregulated or downregulated Lamp2a expression compared with control cells (Fig. 2A). Cell migration as the first step of tumor metastasis is a characteristic of invasive tumors. Wound-healing migration assays were adopted to assess the effect of Lamp2a expression on HCC cell migration. No significant difference 
A
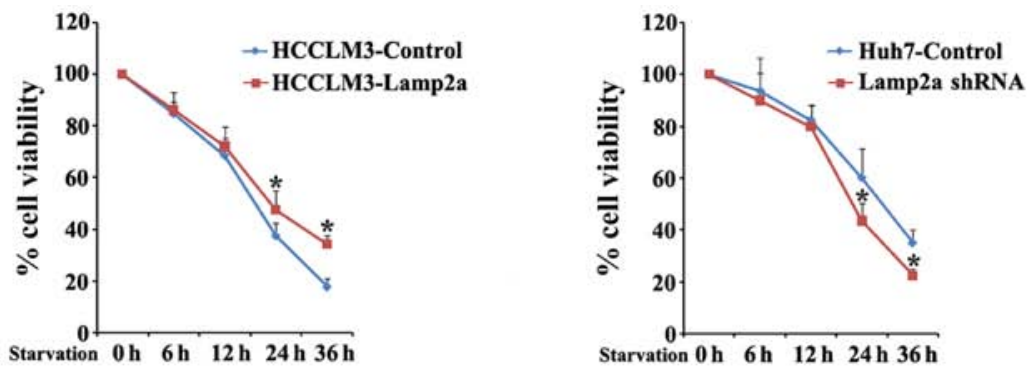

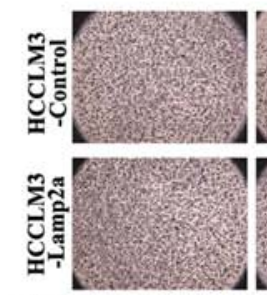

Starvation $\mathbf{0 ~ h}$
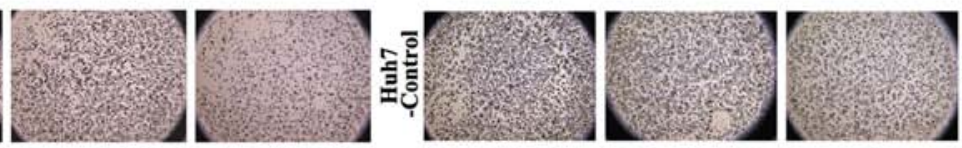

B

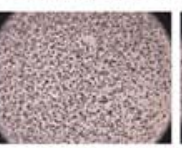

$6 \mathrm{~h}$

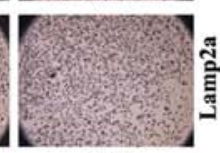

$24 \mathrm{~h}$

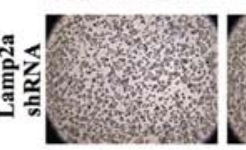

0 h

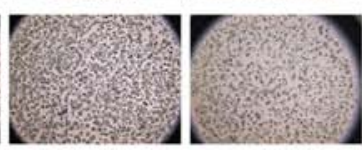

$6 \mathrm{~h}$

24 h
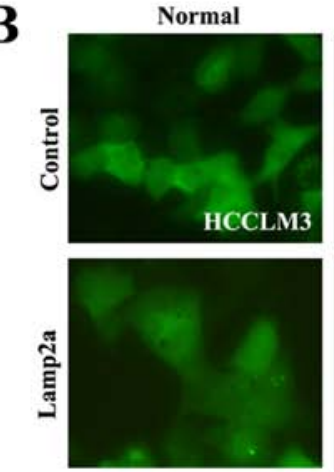

Starvation
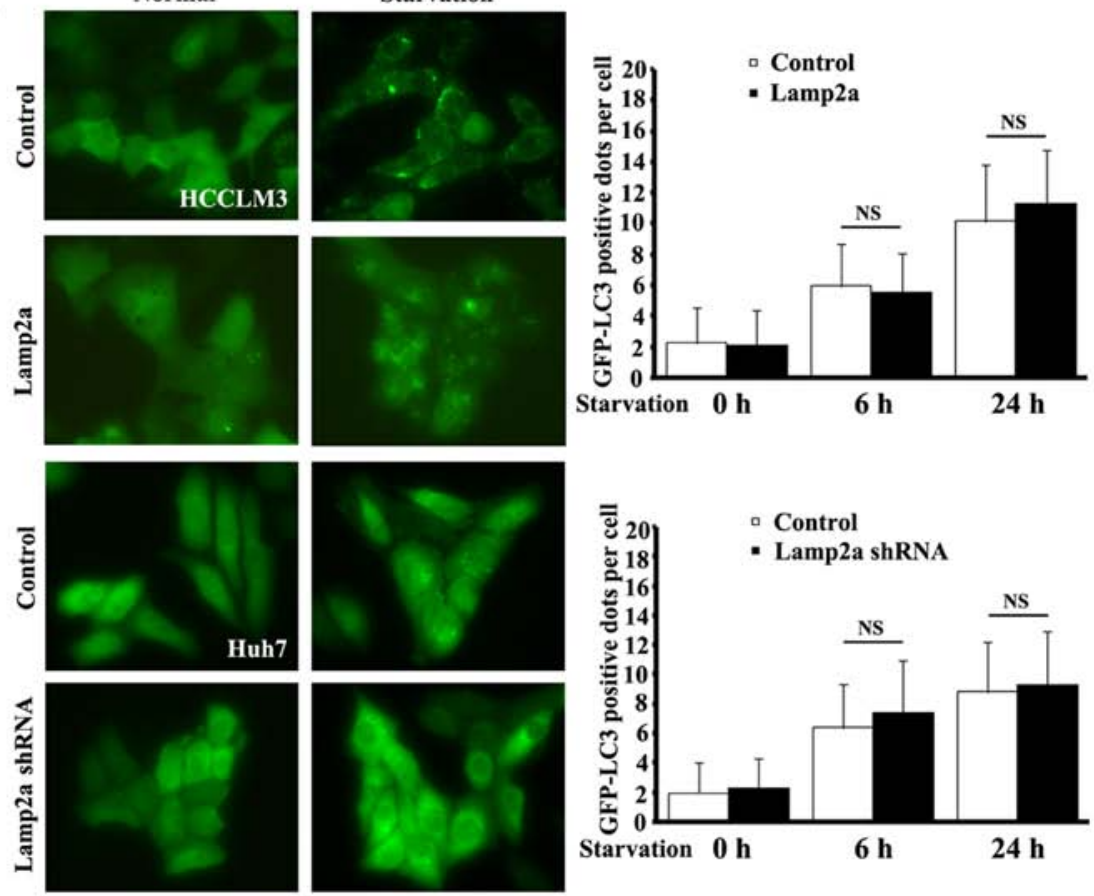

Figure 3. Lamp2a protects HCC cells from prolonged starvation independent of macroautophagy. (A) HCC cell line pairs (HCCLM3-Control/HCCLM3Lamp2a and Huh7-Control/Huh7-Lamp2a shRNA) were incubated in the absence of serum for 6-32 h, measured by absorbance at 570 nm, and plotted as the percentage of the original time-point $(0 \mathrm{~h})$. Data shown are mean (SD) from 3 independent experiments; ${ }^{*} \mathrm{P}<0.05$. (B) HCC cells that stably expressed the GFP-LC3 fusion protein were established. Cells were exposed to starvation for 6 and $24 \mathrm{~h}$, and then observed under the fluorescent microscopy. GFP-LC3positive dots per transfected cell were determined in three independent experiments. Eight random fields representing 200 cells were counted. Data shown are mean (SD). Magnification, x200.

was revealed in wound-closure rate of HCCLM3-Lamp2a and Huh7-Lamp2a shRNA cells compared with the control cells under microscopic examination after 24 and 48 h (Fig. 2B). Invasive capacity of the HCC cells was assessed by Matrigel invasion assays. As shown in Fig. 2C, Lamp2a overexpression in HCCLM3 and Lamp2a silencing in Huh7 cells had no effect on cellular invasion compared with control cells.

Lamp2a protected HCC cells from prolonged starvation. CMA is activated maximally in response to stressors and changes in cellular nutritional status (22). We therefore explored the role of Lamp2a as the critical receptor of CMA in HCC during cell starvation. Isogenic paired HCC cell lines (HCCLM3-Control/HCCLM3-Lamp2a and Huh7-Control/Huh7-Lamp2a shRNA) were incubated in the absence of serum for 6-32 $\mathrm{h}$ and cellular viability was measured using MTT assays (Fig. 3A). There were no differences in viability between the cell line pairs after 6 and $12 \mathrm{~h}$ of starvation, but upregulation of Lamp2a in HCCLM3 cells markedly increased cell survival after prolonged starvation for 24 and $36 \mathrm{~h}$ by 10 and $16.5 \%$, respectively, compared with control cells. Similarly, silencing Lamp2a expression in Huh7-Lamp2a shRNA cells abolished the protective effect of Lamp2a and reduced survival to 16.6 and $12.5 \%$, respectively, compared with control cells.

Macroautophagy is also activated by starvation and is involved in the protective mechanism against environmental 



C
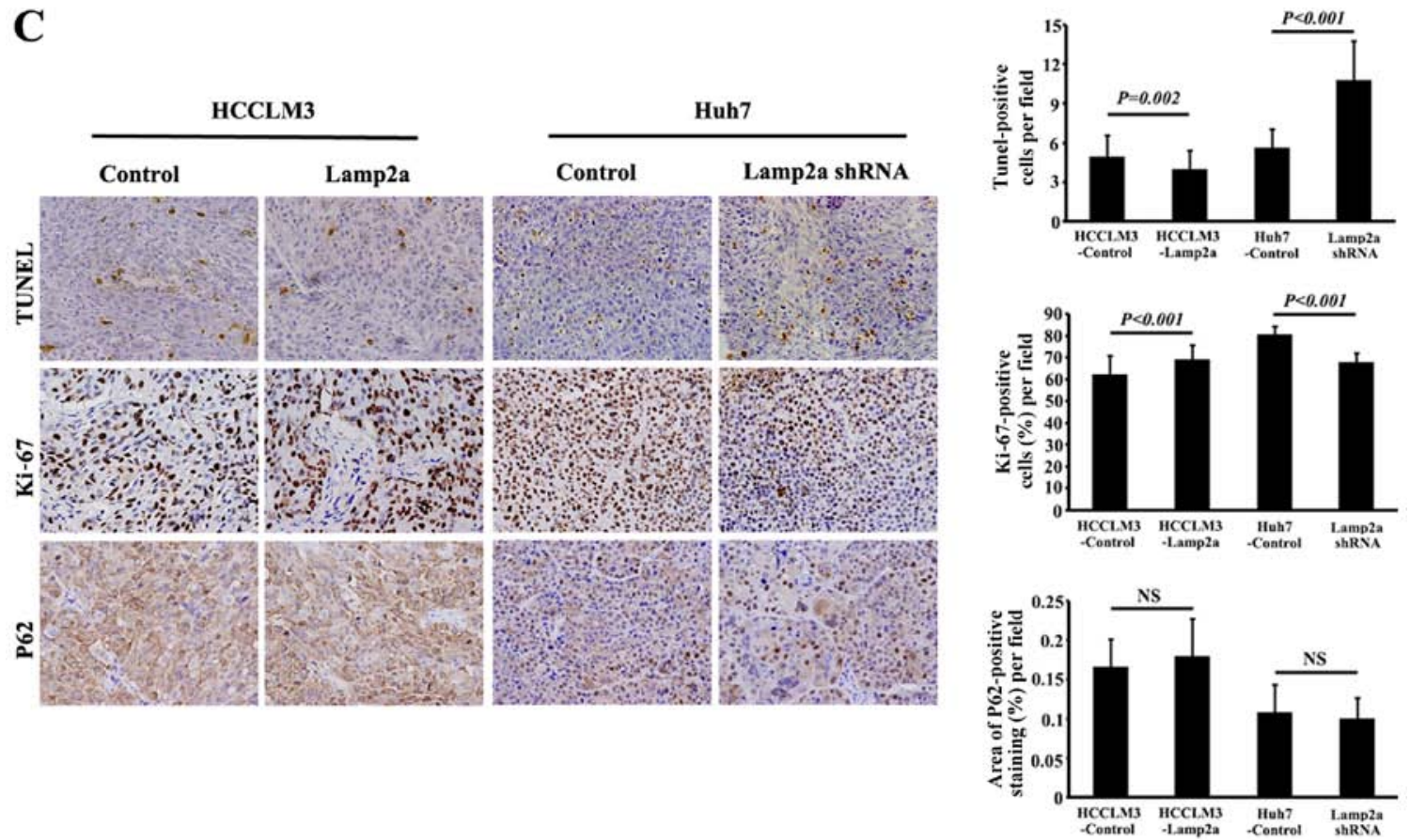

Figure 4. Lamp2a promotes growth of HCC in vivo. (A) Mice bearing HCCLM3-Control/HCCLM3-Lamp2a orthotopic tumors from each group 5 weeks after tumor implantation are shown. Tumor volumes for each group were measured with calipers at 5 weeks. Values are presented as mean (SD). Scale bars, $2 \mathrm{~cm}$. (B) Mice bearing Huh7-Control/Huh7-Lamp2a shRNA subcutaneous tumors from each group 5 weeks after tumor implantation are shown. Tumor volumes for each group were measured with calipers at 5 weeks. Values are presented as mean (SD). Scale bars, $2 \mathrm{~cm}$. (C) Apoptosis was assessed by TUNEL staining, proliferation was assessed by Ki-67 nuclear staining, and autophagy was assessed by P62 staining. TUNEL-positive and Ki-67-positive cells were scored by manual counting in 8 randomly selected fields from each tumor sample. The area of the positive staining of P62 in each photograph was measured by the Image-Pro Plus Software v6.2, and plotted as the percentage of photograph field area. Data shown are means (SD). Magnification, x200. NS, not significant.

and cellular stress (23). We further quantified the level of autophagy by transfecting cells with GFP-LC3 fusion protein (Fig. 3B). Following starvation GFP-LC3 protein changed from a diffuse cytoplasmic distribution to punctate GFP-LC3 dots, indicating a formation of autophagy vacuoles. Morphometric analysis revealed similar numbers of GFP-LC3-positive dots per cell in Lamp2a-modified cells after short and prolonged starvation, indicating that the protection offered by Lamp2a was independent of macroautophagy.
Lamp2a promotes growth of HCC xenograft models. Orthotopic injection of HCCLM3-Control and HCCLM3-Lamp2a, or subcutaneous injection of Huh7-Control and Huh7-Lamp2a shRNA cells in nude mice led to tumor formation in all the groups. However, tumors from HCCLM3-Lamp2a-derived xenografts were significantly larger $\left(2,164.6 \pm 332.0 \mathrm{~mm}^{3}\right)$ than those from HCCLM3-Control xenografts $\left(1,477.6 \pm 732.4 \mathrm{~mm}^{3}\right.$; $\mathrm{P}<0.05$ ) (Fig. 4A). Moreover, Huh7-Lamp2a shRNA-derived xenografts were significantly smaller $\left(426.5 \pm 214.4 \mathrm{~mm}^{3}\right)$ than 
A
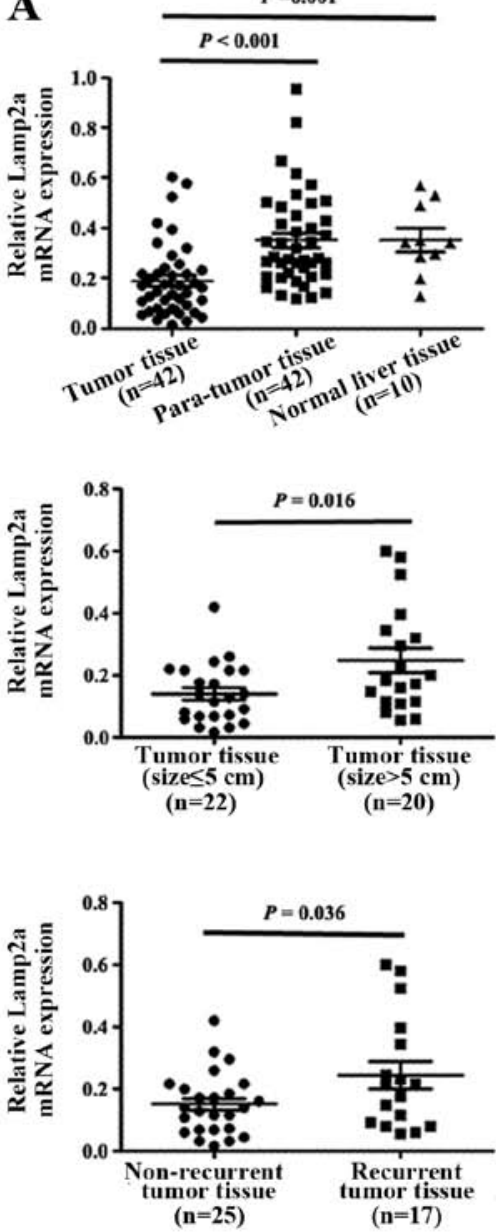

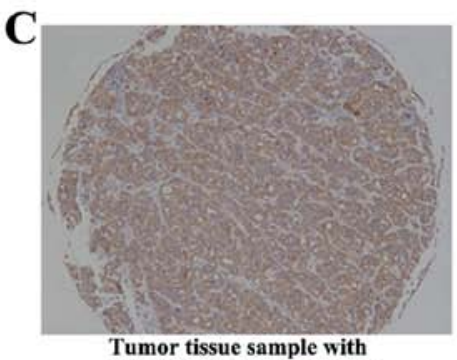

high expression of Lamp2a

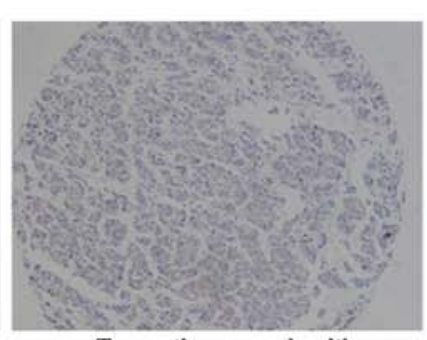

Tumor tissue sample with low expression of Lamp2a
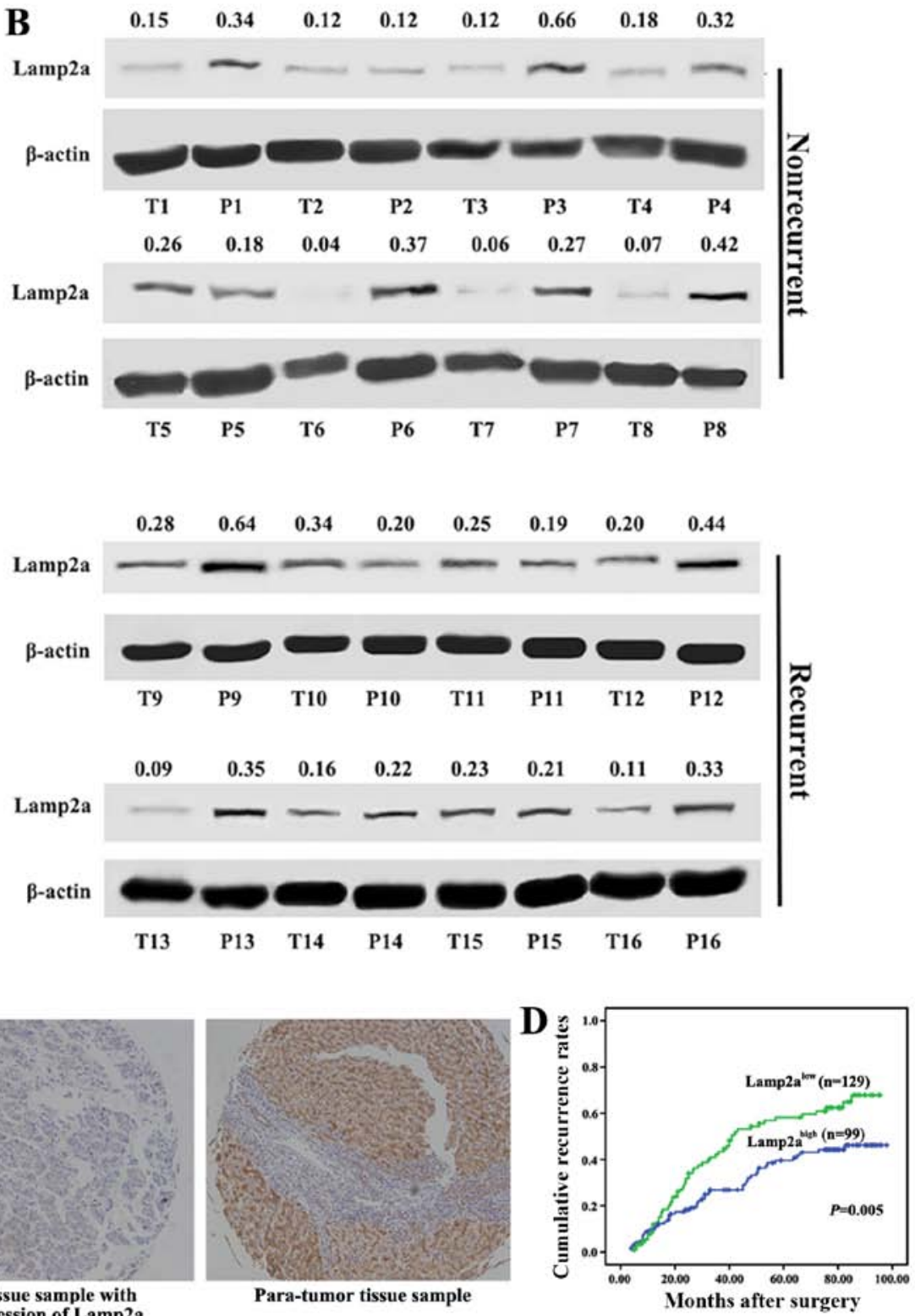

Figure 5. Lamp2a expression in HCC tissue samples and recurrence analysis. (A) Relative Lamp2a mRNA expression in tumor tissue, para-tumor tissue and normal liver tissue (top), small tumor tissue and large tumor tissue (middle), and non-recurrent and recurrent tumor tissue (bottom). Data shown are mean (SD). (B) Lamp2a expression in 16 tumor tissue samples (eight non-recurrent and eight recurrent cases) and corresponding para-tumor tissue samples were confirmed by western blot analysis. (C) Representative immunohistochemical staining of Lamp2a in tumor tissue (positive and negative case, left and middle) and para-tumor tissue (right). Magnification, x200. (D) Kaplan-Meier analysis of cumulative recurrence rates for Lamp2a expression in 228 HCC patients.

Huh7-Control-derived tumors $\left(880.3 \pm 293.3 \mathrm{~mm}^{3} ; \mathrm{P}<0.01\right)$ (Fig. 4B).

Lamp2a overexpression caused a moderate decrease of TUNEL-positive tumor cells compared with HCCLM3Control-derived tumors $(4.0 \pm 1.5$ vs. $4.9 \pm 1.7 ; \mathrm{P}=0.002)$. Significantly more TUNEL-positive tumor cells were also observed in Huh7-shRNA-derived xenografts compared with control tumors (10.7 \pm 3.0 vs. $5.6 \pm 1.4$; $\mathrm{P}<0.001)$ (Fig. 4C). Similarly, Ki-67 staining indicated a significant increase in proliferative activity in the Lamp2a-overexpressing HCCLM3 orthotopic model and control Huh7 subcutaneous model compared with the control HCCLM3 and Huh7-shRNA groups, respectively $(69.0 \pm 6.6$ vs. $62.0 \pm 8.9 \%$; $\mathrm{P}<0.001$, and $80.4 \pm 4.1$ vs. 67.7 $\pm 4.5 \%$; $\mathrm{P}<0.001$ ) (Fig. 4C). We examined the effect of Lamp2a expression on macroautophagy in tumor xenografts in vivo by investigating the expression of $\mathrm{P} 62$, which accumulates during defective autophagy. Immunohistochemical analysis revealed no significant difference of P62 expression between the two groups, suggesting that macroautophagy activity was independent of Lamp2a expression (Fig. 4C).

Lamp2a expression in HCC tissues. We compared Lamp2a mRNA expression in $42 \mathrm{HCC}$ tissue samples and para-tumor tissues and 10 normal livers. Compared with para-tumor tissue samples and normal liver samples, Lamp2a expression was significantly decreased in tumor tissue samples $(\mathrm{P}<0.001$ 
Table I. Correlation between Lamp2a expression and clinicopathological characteristics.

\begin{tabular}{|c|c|c|c|}
\hline \multirow[b]{2}{*}{ Variables } & \multicolumn{2}{|c|}{ Lamp2a expression } & \multirow[b]{2}{*}{ P-value } \\
\hline & $\begin{array}{c}\text { Low } \\
(\mathrm{n}=129)\end{array}$ & $\begin{array}{l}\text { High } \\
(\mathrm{n}=99)\end{array}$ & \\
\hline \multicolumn{4}{|l|}{ Gender } \\
\hline Female & 17 & 10 & \\
\hline Male & 112 & 89 & 0.476 \\
\hline \multicolumn{4}{|c|}{ Age (years) } \\
\hline$\leq 50$ & 54 & 62 & \\
\hline$>50$ & 75 & 37 & 0.002 \\
\hline \multicolumn{4}{|c|}{ Preoperative AFP (ng/ml) } \\
\hline$\leq 20$ & 33 & 45 & \\
\hline$>20$ & 96 & 54 & 0.002 \\
\hline \multicolumn{4}{|c|}{ Liver cirrhosis } \\
\hline No & 34 & 18 & \\
\hline Yes & 95 & 81 & 0.145 \\
\hline \multicolumn{4}{|c|}{ Tumor size (cm) } \\
\hline$\leq 5$ & 85 & 44 & \\
\hline$>5$ & 44 & 55 & 0.001 \\
\hline \multicolumn{4}{|c|}{ Tumor number } \\
\hline Single & 112 & 77 & \\
\hline Multiple & 17 & 22 & 0.072 \\
\hline \multicolumn{4}{|c|}{ Vascular invasion } \\
\hline No & 110 & 91 & \\
\hline Yes & 19 & 8 & 0.124 \\
\hline \multicolumn{4}{|l|}{ Capsule } \\
\hline No & 81 & 64 & \\
\hline Yes & 48 & 35 & 0.773 \\
\hline \multicolumn{4}{|c|}{ Tumor differentiation } \\
\hline I-II & 73 & 61 & \\
\hline III-IV & 56 & 38 & 0.445 \\
\hline
\end{tabular}

AFP, $\alpha$-fetoprotein.

and $\mathrm{P}=0.001$, respectively) (Fig. 5A). Lamp2a expression was lower in $83.3 \%$ (35/42) of tumor tissues compared with matched para-tumor tissues. Levels were decreased $>3$-fold in 10 samples, and 2- to 3 -fold in 11 samples among the above tumor samples. These findings were further confirmed in 16 of the $42 \mathrm{HCC}$ cases by western blot analysis (Fig. 5B). Lamp2a protein levels were decreased in 11 of the 16 samples compared with the para-tumor tissues, especially in cases of non-recurrent HCC. Higher Lamp2a mRNA expression was associated with $\mathrm{HCC}$ recurrence $(\mathrm{P}=0.036)$ and larger HCC size $(\mathrm{P}=0.016)$. Together with the results of the in vivo studies, these findings suggest that Lamp2a expression in HCC tissues contributes to tumor growth and may also influence the clinical prognosis.
We further validated our hypothesis using TMA to evaluate the correlation between Lamp2a and prognosis in $228 \mathrm{HCC}$ patients who underwent curative resection. Strong Lamp2a staining in hepatocytes and tumor cells was observed in most para-tumor tissue samples (176/228) and some tumor tissue samples (47/228) (Fig. 5C). High Lamp2a expression, indicated by moderate or strong immunohistochemical staining in $>20 \%$ of the cells, was present in 43\% (99/228) of HCC patients. However, there was no correlation between Lamp2a expression and clinicopathological characteristics such as gender, liver cirrhosis, tumor number, tumor capsule, tumor differentiation or vascular invasion. Older patients and patients with high $\alpha$-fetoprotein levels had lower Lamp2a expression. Moreover, high expression of Lamp2a was significantly correlated with large tumor size $(\mathrm{P}=0.001)$ (Table I). The overall 3-, 5- and 7-year cumulative recurrence rates in these HCC patients were $34.1,47.9$ and $54.5 \%$, respectively, though patients with high Lamp2a expression had a significantly higher recurrence rate than patients with low Lamp2a expression $(\mathrm{P}=0.005)$ (Fig. 5D). The 3-, 5- and 7-year cumulative recurrence rates for Lamp2 $\mathrm{a}^{\text {high }}$ and Lamp2 $\mathrm{a}^{\text {low }}$ groups were 43.4 vs. $26.8,58.2$ vs. 39.6 and 62.2 vs. $46.2 \%$, respectively.

\section{Discussion}

In contrast to macroautophagy and microautophagy, CMA is a pathway for the selective degradation of individual proteins, mediated by binding to heat shock cognate $70 / 90-\mathrm{kDa}$ protein (HSC70/90) (24), followed by unfolding and translocation of the proteins through the lysosomal membrane by Lamp2a (25). Although both microautophagy and CMA share the HSC70 as a targeting molecule, only CMA depends on Lamp2a (22). The interaction between Lamp2a and substrate proteins is a critical step in the CMA pathway, and changes in the Lamp2a levels of lysosomal membrane therefore modulate the activity (26).

Lamp2a-related CMA activity was shown to decline in the liver of aged mice, and this failure in cellular clearance was proposed to lead to cellular proteotoxicity and accumulation of intracellular damage, ultimately contributing to functional failure in aged organisms $(15,27,28)$. These key observations contribute to a complex but growing convergence between our understandings of the biology of ageing and the mechanisms that underlie cancer (29).

The results of the present study demonstrated that Lamp2a expression was significantly lower in HCC cells compared with the normal hepatic cells. We also compared its mRNA expression levels in HCC, para-tumor and normal liver tissues and, consistent with aged livers in mice, found a significant decrease of Lamp2a expression in HCC tissues compared with para-tumor tissues and normal livers. Furthermore, immunohistochemical analysis of high-throughput TMA showed stronger staining for Lamp2a protein in hepatocytes of para-tumor tissues compared with HCC cells of tumor tissues. These findings suggest that Lamp2a-related CMA may be suppressed in HCC.

Previous studies of CMA activities in breast (14), lung (30) and gastric cancers (31) demonstrated an inconsistent increase in basal CMA activity. Moreover, CMA activation in these cells and tumors was mostly associated with an increase in 
Lamp2a levels. Knock-down of Lamp2a in these cells established that CMA was essential for cancer cell proliferation, tumor growth and metastasis. Lamp2a-related CMA may thus be a tumor-promoter mechanism, and its increased activity may promote human cancer progression.

The results of the present study suggested that Lamp2a expression was downregulated in HCC tissues, but the function of the remaining Lamp2a protein in HCC is still unclear. Gain and loss of Lamp2a function were explored in HCCLM3 and Huh7 HCC cell lines, respectively. HCC cell lines with stable upregulation or downregulation of Lamp2a expression were confirmed by western blotting, but no differences in cell morphology or function were observed between the two groups under normal culture conditions. However, autophagy induces survival mechanisms that prevent against tumor cell death during hypoxia, starvation and oxidative/metabolic stress (32). We therefore observed cellular viability during starvation for 6-36 h, and found no effect of Lamp2a expression on cell viability during short-term starvation, but Lamp2a blockage significantly inhibited HCC cell survival under prolonged starvation. These results suggest that Lamp2amediated CMA activation may promote long-term cancer cell survival. Moreover, Lamp2a overexpression induced HCC xenograft growth in vivo. Downregulation of Lamp2a resulted in a marked increase in TUNEL-positive tumor cells and a decrease in Ki-67 staining, suggesting that Lamp2a aids tumor growth by helping cells to avoid apoptosis and promoting cell proliferation.

The present study raises the question of why impaired Lamp2a expression increased HCC tumor survival under stress. We speculate that, similar to macroautophagy $(33,34)$, Lamp2a-related CMA may have an opposing effect, via the degradation of modified and oxidatively damaged proteins (35). Reduced CMA activity in aged organisms contributes to the proteins aggregation and abnormal intracellular homeostasis $(15,27,28)$. Lamp2a defects at an early stage of oncogenesis may thus accelerate HCC tumorigenesis as a result of reduced CMA activity and abnormal protein degradation. However, once the tumor is formed, Lamp2a-related CMA acts as part of a stress-response mechanism to protect the cancer cells against oxidative insults or low nutrient supply.

As a result of high recurrence rate after surgery and development of tumors in intrahepatic metastases or cirrhotic liver, the prognosis of $\mathrm{HCC}$ is poor (36). As a critical receptor for CMA, Lamp2a may play a role in tumor growth, ultimately contributing to the poor prognosis. These real-time PCR and immunohistochemical analyses of HCC tissue samples supported a strong correlation between Lamp2a expression and tumor size, while 3-, 5- and 7-year cumulative recurrence rates were also significantly lower in patients with higher Lamp2a expression levels. To the best of our knowledge, this study is the first to report Lamp2a-mediated tumor survival and recurrence in human $\mathrm{HCC}$.

In conclusion, impaired Lamp2a expression in HCC is required for tumor growth by enabling cells to avoid apoptosis and by promoting cell proliferation. Lamp2a may serve as a predictive marker for $\mathrm{HCC}$ viability and recurrence, indicating the need for more aggressive treatment. Targeting chaperonemediated autophagy through Lamp2a may also represent a potentially novel therapeutic strategy for HCC.

\section{Acknowledgements}

The present study was supported by the funds from the National Natural Science Fund of China (nos. 81472219 and 81602037).

\section{References}

1. Torre LA, Bray F, Siegel RL, Ferlay J, Lortet-Tieulent J and Jemal A: Global cancer statistics, 2012. CA Cancer J Clin 65: 87-108, 2015

2. Amaravadi RK and Thompson CB: The roles of therapy-induced autophagy and necrosis in cancer treatment. Clin Cancer Res 13: 7271-7279, 2007.

3. Kondo Y, Kanzawa T, Sawaya R and Kondo S: The role of autophagy in cancer development and response to therapy. Nat Rev Cancer 5: 726-734, 2005.

4. Lee YJ and Jang BK: The role of autophagy in hepatocellular carcinoma. Int J Mol Sci 16: 26629-26643, 2015.

5. Amaravadi RK, Lippincott-Schwartz J, Yin XM, Weiss WA, Takebe N, Timmer W, DiPaola RS, Lotze MT and White E: Principles and current strategies for targeting autophagy for cancer treatment. Clin Cancer Res 17: 654-666, 2011.

6. Chiang HL and Dice JF: Peptide sequences that target proteins for enhanced degradation during serum withdrawal. J Biol Chem 263: 6797-6805, 1988.

7. Xilouri M, Brekk OR, Kirik D and Stefanis L: LAMP2A as a therapeutic target in Parkinson disease. Autophagy 9: 2166-2168, 2013.

8. Fidziańska A, Walczak E and Walski M: Abnormal chaperonemediated autophagy (CMA) in cardiomyocytes of a boy with Danon disease. Folia Neuropathol 45: 133-139, 2007.

9. Venugopal B, Mesires NT, Kennedy JC, Curcio-Morelli C, Laplante JM, Dice JF and Slaugenhaupt SA: Chaperonemediated autophagy is defective in mucolipidosis type IV. J Cell Physiol 219: 344-353, 2009.

10. Cuervo AM and Dice JF: Regulation of lamp2a levels in the lysosomal membrane. Traffic 1: 570-583, 2000.

11. Cuervo AM, Knecht E, Terlecky SR and Dice JF: Activation of a selective pathway of lysosomal proteolysis in rat liver by prolonged starvation. Am J Physiol 269: C1200-C1208, 1995.

12. Cuervo AM and Dice JF: Unique properties of lamp2a compared to other lamp2 isoforms. J Cell Sci 113: 4441-4450, 2000.

13. Park Y, Liu C, Luo T, Dietrich WD, Bramlett H and Hu B: Chaperone-mediated autophagy after traumatic brain injury. $\mathrm{J}$ Neurotrauma 32: 1449-1457, 2015.

14. Saha T: LAMP2A overexpression in breast tumors promotes cancer cell survival via chaperone-mediated autophagy. Autophagy 8: 1643-1656, 2012.

15. Zhang C and Cuervo AM: Restoration of chaperone-mediated autophagy in aging liver improves cellular maintenance and hepatic function. Nat Med 14: 959-965, 2008.

16. Ding ZB, Shi YH, Zhou J, Qiu SJ, Xu Y, Dai Z, Shi GM, Wang XY, Ke AW, Wu B, et al: Association of autophagy defect with a malignant phenotype and poor prognosis of hepatocellular carcinoma. Cancer Res 68: 9167-9175, 2008.

17. Hui B, Shi YH, Ding ZB, Zhou J, Gu CY, Peng YF, Yang H, Liu WR, Shi GM and Fan J: Proteasome inhibitor interacts synergistically with autophagy inhibitor to suppress proliferation and induce apoptosis in hepatocellular carcinoma. Cancer 118: 5560-5571, 2012.

18. Ding ZB, Hui B, Shi YH, Zhou J, Peng YF, Gu CY, Yang H, Shi GM, Ke AW, Wang XY, et al: Autophagy activation in hepatocellular carcinoma contributes to the tolerance of oxaliplatin via reactive oxygen species modulation. Clin Cancer Res 17: 6229-6238, 2011 .

19. Shi YH, Ding ZB, Zhou J, Hui B, Shi GM, Ke AW, Wang XY, Dai Z, Peng YF, Gu CY, et al: Targeting autophagy enhances sorafenib lethality for hepatocellular carcinoma via ER stressrelated apoptosis. Autophagy 7: 1159-1172, 2011.

20. Peng YF, Shi YH, Ding ZB, Ke AW, Gu CY, Hui B, Zhou J, Qiu SJ, Dai $Z$ and Fan J: Autophagy inhibition suppresses pulmonary metastasis of HCC in mice via impairing anoikis resistance and colonization of HCC cells. Autophagy 9: 2056-2068, 2013.

21. Gao Q, Qiu SJ, Fan J, Zhou J, Wang XY, Xiao YS, Xu Y, Li YW and Tang ZY: Intratumoral balance of regulatory and cytotoxic $\mathrm{T}$ cells is associated with prognosis of hepatocellular carcinoma after resection. J Clin Oncol 25: 2586-2593, 2007. 
22. Kaushik S, Bandyopadhyay U, Sridhar S, Kiffin R, MartinezVicente M, Kon M, Orenstein SJ, Wong E and Cuervo AM: Chaperone-mediated autophagy at a glance. J Cell Sci 124: 495-499, 2011.

23. Lebovitz CB, Bortnik SB and Gorski SM: Here, there be dragons: Charting autophagy-related alterations in human tumors. Clin Cancer Res 18: 1214-1226, 2012.

24. Chiang HL, Terlecky SR, Plant CP and Dice JF: A role for a 70-kilodalton heat shock protein in lysosomal degradation of intracellular proteins. Science 246: 382-385, 1989.

25. Cuervo AM and Dice JF: A receptor for the selective uptake and degradation of proteins by lysosomes. Science 273: 501-503, 1996.

26. Massey AC, Kaushik S, Sovak G, Kiffin R and Cuervo AM: Consequences of the selective blockage of chaperone-mediated autophagy. Proc Natl Acad Sci USA 103: 5805-5810, 2006.

27. Schneider JL, Villarroya J, Diaz-Carretero A, Patel B Urbanska AM, Thi MM, Villarroya F, Santambrogio L and Cuervo AM: Loss of hepatic chaperone-mediated autophagy accelerates proteostasis failure in aging. Aging Cell 14: 249-264, 2015.

28. Schneider JL, Suh Y and Cuervo AM: Deficient chaperonemediated autophagy in liver leads to metabolic dysregulation. Cell Metab 20: 417-432, 2014.
29. Finkel T, Serrano M and Blasco MA: The common biology of cancer and ageing. Nature 448: 767-774, 2007.

30. Kon M, Kiffin R, Koga H, Chapochnick J, Macian F, Varticovski L and Cuervo AM: Chaperone-mediated autophagy is required for tumor growth. Sci Transl Med 3: 109ra117, 2011.

31. Zhou J, Yang J, Fan X, Hu S, Zhou F, Dong J, Zhang S, Shang Y, Jiang $\mathrm{X}$, Guo $\mathrm{H}$, et al: Chaperone-mediated autophagy regulates proliferation by targeting RND3 in gastric cancer. Autophagy 12: 515-528, 2016.

32. Hait WN, Jin S and Yang JM: A matter of life or death (or both): Understanding autophagy in cancer. Clin Cancer Res 12: 1961-1965, 2006.

33. White E and DiPaola RS: The double-edged sword of autophagy modulation in cancer. Clin Cancer Res 15: 5308-5316, 2009.

34. Cicchini M, Karantza V and Xia B: Molecular pathways: Autophagy in cancer - a matter of timing and context. Clin Cancer Res 21: 498-504, 2015.

35. Hubbi ME, Hu H, Kshitiz, Ahmed I, Levchenko A and Semenza GL: Chaperone-mediated autophagy targets hypoxiainducible factor- $1 \alpha(\mathrm{HIF}-1 \alpha)$ for lysosomal degradation. J Biol Chem 288: 10703-10714, 2013.

36. Sherman M: Recurrence of hepatocellular carcinoma. N Engl J Med 359: 2045-2047, 2008. 